\title{
A new platform for drug discovery
}

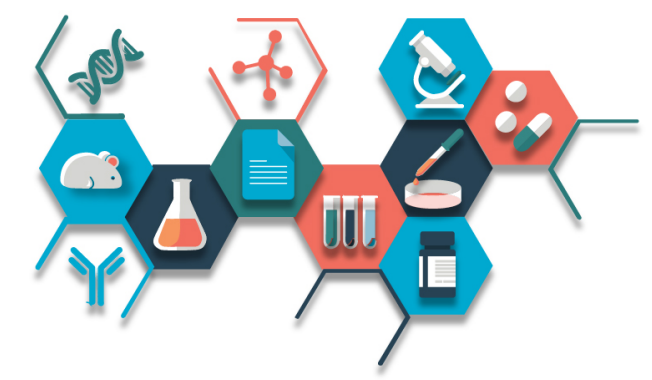

Tuomas PJ Knowles*,1

${ }^{1}$ Fluidic Analytics, Unit A, The Paddocks Business Centre, Cherry Hinton Road, Cambridge, CB1 8DH, UK

*Author for correspondence: tpjk2@cam.ac.uk

Professor Tuomas Knowles gained his PhD in biophysics from the University of Cambridge (UK) in 2007 and went on to work at Harvard University (MA, USA) before returning to Cambridge as a lecturer, gaining professorship in 2015. He is the founder and Chief Scientific Officer of Fluidic Analytics (Cambridge, UK), a biotech company developing next-generation protein analysis platforms that operate under native conditions in solution.

Here he speaks to Future Drug Discovery Editor Jennifer Straiton about Fluidic Analytics' new platform Fluidity One-W, discussing how it works and what benefit it can bring to the field of drug discovery.

First draft submitted: 6 September 2019; Accepted for publication: 16 September 2019; Published online: 7 October 2019

\section{Please could you introduce us to Fluidity One-W?}

Within Fluidic Analytics, we are trying to develop new ways of characterizing proteins and, in particular, we are developing a platform that allows proteins and protein-protein interactions to be studied under native conditions. In many conventional approaches for studying proteins and their interactions, one of the interaction partners is typically either attached to a surface, for example, in immunoassays or biosensing, or the system to be studied is embedded in a matrix, such as a gel, to allow for separation between bound and unbound species. These are the two types of strategies that are currently used and while they do work, they involve probing proteins under conditions that are not their native solution environment. At Fluidic Analytics, we are working on developing practical ways to allow experiments of this type to be done but without having to attach proteins to surfaces or embed them into gels. This gives us the ability to work with more complex, challenging targets and look at highly dynamic systems on a wide range of time scales. With this as our general strategy, we are bringing out this technology to the protein science community by releasing a series of specific instruments each with a different role. Our most recent release is the Fluidity One-W, which is a specific instrument for detecting and characterizing protein-protein interactions.

\section{How does Fluidity One-W work?}

In principle, the way that we achieve the characterization of proteins in solution is by studying their mobility, their diffusion behavior, and we do this in a way that exploits fluid behavior on very small scales in a process termed diffusional sizing. On these small scales within our microfluidic devices there is no convective mixing; what that means is that the fluid flow is fully laminar which is unlike fluid behavior on a large scale. On a larger scale, fluids typically like to mix; however, that feature disappears when you make the volumes small enough and then it gets to the point when even perfectly miscible fluids will just very happily sit next to each other without actually mixing. This gives a highly controlled environment which can be exploited in the study of the behavior of biomolecules. The way that Fluidity One-W works is that we bring together two fluids, one containing the molecule of interest and the other simply a buffer. We then look at how rapidly the molecules move from one side to the other. The idea is that we can highlight one molecule of interest by attaching a label and then track this molecule to see whether it is interacting with a partner. If it is then its diffusion coefficient will change; its diffusion coefficient depends on the molecular weight and the size and when we have interactions, we can pick this up quantitatively based on the change in the diffusion coefficient.

\section{What makes the Fluidity One-W different to existing options?}

Fundamentally what this instrument allows us to do is characterize proteins completely under native conditions. This feature removes the requirement to functionalize the surface to enhance the adhesion of some partners and prevent the adhesion of others. A big factor in immunoassays is having to selectively functionalize a surface and 
then passivate the rest of the surface with various treatments; this is something we just don't have to do with Fluidity One-W. It is much faster and we can just deposit the drop onto the chip, put the chip into the machine and the reading comes out in a matter of minutes.

The second feature is that it allows us to work with more complex targets which can be highly challenging to attach to surfaces or to run on gels. We are very excited about looking at intrinsically disordered proteins as these do not have a well-defined globular structure, are increasingly recognized as core hub proteins in many signaling networks and have structures that are very prone to be perturbed if attached onto surfaces. We are also interested in looking at aggregated proteins which are highly heterogeneous and very difficult to run on gels or attach to surfaces in a controlled way.

\section{Are there any limitations with this technique or challenges still to overcome with this technology?}

There are always challenges - the challenges that we have had to overcome recently have been largely engineering related; in order to make the technology easy to use and to make it accessible there is a lot of engineering work. Going forward, one of the challenges we are actively working on is measuring different physical properties other than just size, characterizing proteins in a multidimensional way. We are also looking at increasing the resolution, allowing us to resolve particular components in a highly complex mixture.

\section{How can this be best utilized for drug discovery?}

On a molecular level, a lot of drug discovery is looking for interactions, so we think that this is a very general platform to be able to detect and screen for interactions. There are a number of areas where this native solution diffusional sizing technology offers advantages, such as those I mentioned earlier.

In the case of intrinsically disordered proteins, which are emerging as interesting targets, probing using existing methods is challenging, due to the disorder, which makes them very sensitive to being attached to surfaces or embedded into gels. However, about $20-30 \%$ of human proteins are either disordered or have a significant portion of them that has disorder, so it is not a rare phenomenon and is a key part of many interesting proteins.

We are also interested in making our technology available for researchers working on studying protein aggregates, which are involved in many disorders but that have been historically challenging targets because these systems are very highly heterogeneous and are highly dynamic. These are exactly the kind of features you do not want when using conventional methods. What we find is in our native solution microfluidic approach it does not really matter as we get equally good binding curves for these kinds of proteins. Together with colleagues from the University of Cambridge, ETH Zurich (Switzerland), the University of Wollongong (Australia) and Lund University (Sweden), we recently published a paper in Science Advances [1] where we demonstrated the power of this approach for probing protein aggregates. In the paper, rather than looking at drug molecules, we looked at naturally occurring protective molecules called molecular chaperones, which have the ability to recognize protein aggregates. These are natural molecules expressed in the cell which protect us against the deleterious effects of protein aggregates. We wanted to try and understand how they bind to protein aggregates, how they recognize them and where they bind. These are questions which have been very challenging to address via conventional methods and we have found that by using diffusional sizing we were able to study these highly complex systems in a very quantitative way, measuring binding constants and binding kinetics.

An area that we are very interested in is antibody characterization, that is understanding the stability of antibodies and the properties that determine their specificity and selectivity. These are all characteristics where being able to do measurements under native conditions in solution is going to be much closer to the in vivo situation than trying to do these on surface-based assays.

These are the three areas that we are currently exploring and are very excited about, but in principle we believe that in any screening activity where one or more of the molecules to be studied are proteins, we can provide an attractive way forward. In particular, to date, a lot of drug discovery has focused on small molecule-protein interactions and modulating protein-protein interactions has remained more challenging. Measuring these interactions in the first place is not so easy with conventional technology, especially if one of the partners is disordered and we are very excited about doing this using our platform. 


\section{What is next for this technology?}

Right now, we have this platform technology and we are bringing out individual products to address specific problems. The Fluidity One-W is specifically a product to look at protein-protein interactions so one of the things we are working on is to make a more high-throughput version. We are also working on measuring physical properties other than just size and molecular weight. We are very interested in charge as well; these are the two areas we are working in: providing more granular information and increasing the throughput of the platform.

\section{Do you see this being used in all aspects of bioanalysis, not just drug discovery?}

We are very excited about the technology and we think if you have the choice between characterizing proteins under native conditions or non-native conditions there is no real reason not to use native conditions; they will more closely mimic the protein behavior in the real biological system and so we think the potential here is great. We do think drug discovery is a very exciting and very important area, and so we are very keen to provide instrumentation to make an impact in that space, but the technology has more general applications as both a lab tool and also in diagnostics. I think linking protein science to biomedical problems is a really exciting area overall and we are really looking forward to bringing fundamentally new technology to an area which so far has rather relied on traditional methods.

\section{Financial \& competing interests disclosure}

The authors have no other relevant affiliations or financial involvement with any organization or entity with a financial interest in or financial conflict with the subject matter or materials discussed in the manuscript apart from those disclosed.

The opinions expressed in this interview are those of TPJ Knowles and do not necessarily reflect the views of Future Science Ltd.

\section{Open access}

This work is licensed under the Attribution-NonCommercial-NoDerivatives 4.0 Unported License. To view a copy of this license, visit http://creativecommons.org/licenses/by-nc-nd/4.0/

\section{References}

1. Scheidt T, Łapińska U, Kumita JR et al. Secondary nucleation and elongation occur at different sites on Alzheimer's amyloid- $\beta$ aggregates. Sci. Adv. 5(4), eaau3112 (2019). 